\title{
Binaries in the Hyades
}

\author{
ROBERT P. STEFANIK \& DAVID W. LATHAM \\ Harvard-Smithsonian Center for Astrophysics, 60 Garden Street, \\ Cambridge, MA 02138, USA
}

\begin{abstract}
Orbital solutions are now available for 46 binaries which are members of the Hyades. The distribution of eccentricity versus period shows evidence for tidal circularization of the short-period binaries. However, the transition from circular to eccentric orbits is not clean. The first eccentric orbit has a period of 5.75 days, while the last circular orbit has a period of 8.50 days. For longer periods the distribution of eccentricity is the same as for solar-type stars in the field.
\end{abstract}

In Table 1 we list the period and eccentricity for 46 binaries which are members of the Hyades star cluster and have reliable orbits. Six of the binaries have visual orbits, two have orbits incorporating speckle and/or occultation observations, and two have orbits combining radial velocities with astrometric information (indicating that the traditional gap between astrometric and spectroscopic orbits is closing). Altogether 36 Hyades binaries have spectroscopic orbits, of which 12 are new or revised orbits using CfA radial velocities. In the CfA sample of slightly more than 200 Hyades members, we have at least 15 additional spectroscopic binaries which do not yet yield satisfactory orbital solutions, but which clearly exhibit velocity variations.

In Figure 1 we plot eccentricity versus log period for all 46 Hyades binaries that have orbital solutions. All the binaries with periods shorter than 5.75 days have circular orbits, presumably due to tidal circularization (e.g., see Mathieu et al. 1992). However, the transition from circular to eccentric orbits is not clean. The last circular orbit has a period of 8.50 days, substantially longer than the period of 5.75 days for the first eccentric orbit. It is interesting to note that the spectral types are classified as F8 for the primary stars in the two shortestperiod binaries ( 5.75 and 8.55 days) with eccentric orbits. These primaries are close to the dividing line where more massive stars no longer have significant convective zones, and tidal circularization becomes much less effective (e.g., see Mathieu \& Mazeh 1988).

Binaries which are members of triple systems are plotted as open circles in Figure 1. The small but significant eccentricity of the short-period members of triples is presumably the result of the pumping action of the distant third star (Mazeh 1990).

The mean eccentricity for the 30 main-sequence Hyades binaries with orbital period longer than 10 days is $0.45 \pm 0.04$. This is very similar to the mean eccentricity of $0.42 \pm 0.03$ for a sample of 44 binaries with period longer than 14 days found among 164 nearby solar-type field dwarfs (Duquennoy \& Mayor 1991). 


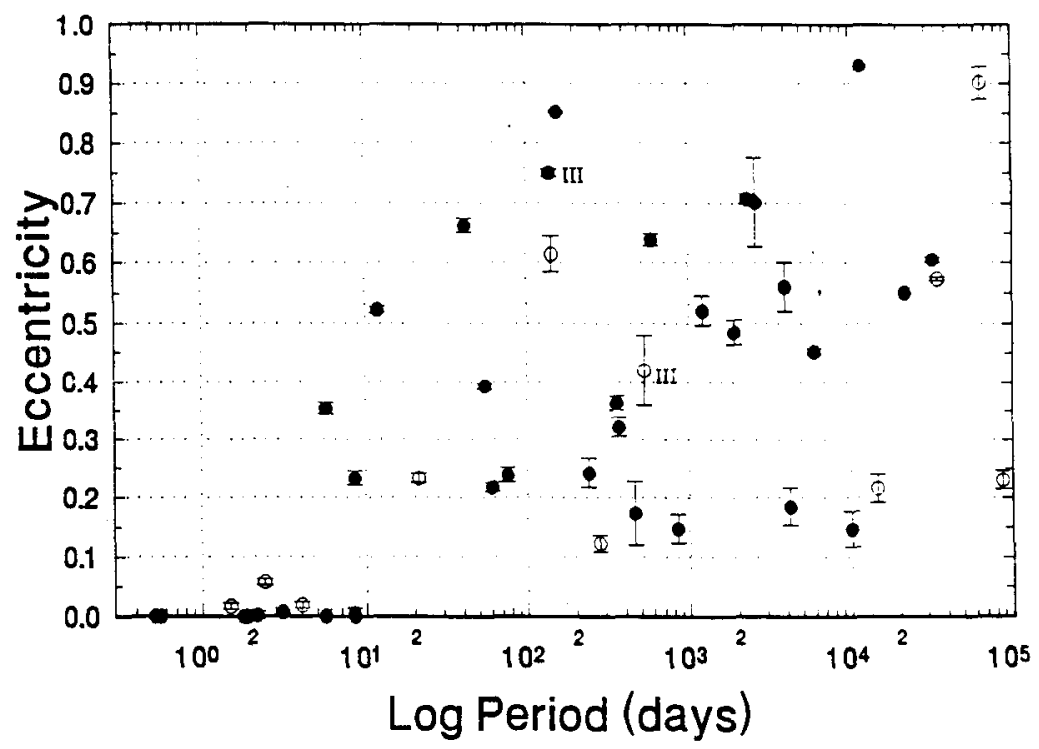

FIGURE 1. Eccentricity versus $\log$ period for 46 binaries in the Hyades.

We thank Ed Horine, Jim Peters, Joe Caruso, and Joe Zajac for making many of the observations; Bob Davis for help with the observing and data reduction; and Roger Griffin for suggesting the correct period for HD 286839. SIMBAD was an essential tool in our search for published orbital solutions. Limited space did not allow us to include proper references and notes for Table 1. They will be published in a forthcoming paper.

\section{REFERENCES}

Duquennoy, A. \& Mayor, M. 1991, $A \& A, \mathbf{2 4 8}, 485$

Mathieu, R.D., Duquennoy, A., Latham, D.W., Mayor, M., Mazeh, T., \& Mermilliod, J.-C. 1992, in Workshop on Binaries as Tracers of Stellar Formation and Evolution, eds. A. Duquennoy and M. Mayor (Cambridge, Cambridge University Press), in press

Mathieu, R.D. \& Mazeh, T. 1988, ApJ, 326, 256

Mazeh, T. 1990, $A J, 99,675$ 
TABLE 1. Hyades binaries.

\begin{tabular}{|c|c|c|c|c|c|c|}
\hline Name & $V$ & $B-V$ & Spectrum & Period & Eccentricity & Reference \\
\hline HD 28319 & 3.40 & 0.18 & A7III & 140.728 & $0.750 \pm 0.006$ & Eb59 \\
\hline HD 27176 & 5.65 & 0.28 & $\mathrm{~A} 8 \mathrm{~V}+\mathrm{G} 0$ & $4,177.31$ & $0.185 \pm 0.031$ & CfA \\
\hline HZ 9 & 14.02 & 0.29 & $\mathrm{DA}+\mathrm{dM} 4.5$ & 0.564 & 0.0 & L\&P81 \\
\hline HD 27749 & 5.64 & 0.30 & Alm & 8.418 & $0.005 \pm 0.008$ & CfA \\
\hline HD 27628 & 5.72 & 0.32 & A3m & 2.144 & $0.001 \pm 0.006$ & CfA \\
\hline HD 28485 & 5.58 & 0.32 & $A 8 V+G 2 V$ & $64,977.98$ & $0.901 \pm 0.027$ & P\&S88 \\
\hline HD 27483 & 6.17 & 0.46 & F6V & 3.059 & $0.007 \pm 0.005$ & CfA \\
\hline HD 30869 AB & 6.25 & 0.49 & F5V & $34,786.41$ & $0.573 \pm 0.003$ & P\&S88 \\
\hline HD $30869 \mathrm{~A}$ & 6.25 & 0.49 & F5V & 143.53 & $0.614 \pm 0.030$ & GGZG85 \\
\hline HD 27991 & 6.46 & 0.49 & F7V & $2,294.14$ & $0.707 \pm 0.009$ & M88 \\
\hline HD 30738 & 7.29 & 0.50 & F8 & 5.751 & $0.354 \pm 0.010$ & G\&G78 \\
\hline HD 28394 & 7.05 & 0.50 & F7V & 238.87 & $0.242 \pm 0.025$ & GGZG85 \\
\hline HD $28363 \mathrm{AB}$ & 6.59 & 0.53 & F7V+G0V & $14,584.43$ & $0.216 \pm 0.024$ & P\&S88 \\
\hline HD $28363 \mathrm{~A}$ & 6.59 & 0.53 & F7V+GOV & 21.254 & $0.233 \pm 0.008$ & CfA \\
\hline HD 28033 & 7.38 & 0.54 & F8V & 8.551 & $0.233 \pm 0.012$ & G\&G78 \\
\hline HD 30810 & 6.76 & 0.54 & F8V & $5,946.75$ & $0.453 \pm 0.005$ & P\&S88 \\
\hline HD $27691 \mathrm{~A}$ & 6.99 & 0.56 & G1 & 4.000 & $0.018 \pm 0.005$ & $\mathrm{CfA}$ \\
\hline HD $27691 \mathrm{AB}$ & 6.99 & 0.56 & F8IV+G1 & $87,331.28$ & $0.230 \pm 0.016$ & P\&S88 \\
\hline HD 27383 & 6.88 & 0.56 & F7V + G3V V & $32,594.91$ & $0.605 \pm 0.004$ & P\&S88 \\
\hline HD 28068 & 8.08 & 0.64 & GiV & $2,595.3$ & $0.701 \pm 0.075$ & CfA \\
\hline HD 26090 & 8.33 & 0.64 & G1V+G8V & 21,915 & 0.55 & M78 \\
\hline HD 33204 B & 8.54 & 0.67 & dG7 & $11,724.5$ & 0.93 & H76 \\
\hline HD 27989 & 7.53 & 0.68 & G3V+G6V & $10,077.25$ & $0.147 \pm 0.030$ & P\&S88 \\
\hline HD 27149 & 7.54 & 0.68 & G5V & 75.657 & $0.239 \pm 0.012$ & P\&S88 \\
\hline HD 26874 & 7.84 & 0.71 & G4V & $\mathbf{5 5 . 1 3 0}$ & $0.393 \pm 0.005$ & G\&G81 \\
\hline HD 28291 & 8.64 & 0.75 & G8V & 41.663 & $0.662 \pm 0.012$ & GGZG85 \\
\hline HD 27935 & 8.94 & 0.76 & G8V & 156.387 & $0.852 \pm 0.001$ & GGZG85 \\
\hline HD 27130 & 8.34 & 0.77 & G8V & 5.609 & 0.0 & P\&S88 \\
\hline HD 28545 & 8.93 & 0.85 & G8V & 358.41 & $0.364 \pm 0.012$ & G\&G81 \\
\hline$+16^{\circ} 516$ & 9.51 & 0.85 & $\mathrm{DA}+\mathrm{K} 2 \mathrm{~V}$ & 0.521 & 0.0 & BLM88 \\
\hline HD 284414 & 9.40 & 0.91 & $\mathrm{~K} 2 \mathrm{~V}$ & 590.6 & $0.638 \pm 0.010$ & GGZG85 \\
\hline HD 27697 & 3.76 & 0.98 & KOIII & 529.8 & $0.42 \pm 0.06$ & G\&G77 \\
\hline HD 284303 & 9.52 & 0.98 & Ko & 1.887 & 0.0 & GMG82 \\
\hline HD 28634 & 9.53 & 0.98 & $\mathrm{~K} 2$ & 844.6 & $0.148 \pm 0.024$ & GGZG85 \\
\hline HD 29896 & 9.87 & 0.99 & KO & 3,942 . & $0.56 \pm 0.04$ & GGZG85 \\
\hline HD 283882 & 9.54 & 1.05 & $\mathbf{K} 3 \mathbf{V}$ & 11.924 & $0.523 \pm 0.005$ & CfA \\
\hline HD 285766 & 10.16 & 1.06 & $\mathrm{~K} 2$ & 1,907 & $0.485 \pm 0.021$ & GGZG85 \\
\hline BD 16909 & 8.17 & 1.07 & $\mathrm{dK} 4$ & 1,214 & $0.521 \pm 0.024$ & GGZG85 \\
\hline HD 284163 & 9.38 & 1.09 & KO & 2.394 & $0.057 \pm 0.005$ & G\&G81 \\
\hline HD 285828 & 10.39 & 1.09 & $\mathbf{K} 2$ & 459.197 & $0.174 \pm 0.054$ & CEA \\
\hline HD 29608 & 9.47 & 1.10 & $\mathbf{K} 3 \mathbf{V}$ & 276.76 & $0.123 \pm 0.014$ & GGZG85 \\
\hline HD 283750 & 8.25 & 1.12 & $\mathrm{dK}$ & 1.788 & 0.0 & GGZG85 \\
\hline HD 285947 & 10.17 & 1.15 & K5 & 60.821 & $0.218 \pm 0.008$ & GGZG85 \\
\hline HD 286839 & 11.03 & 1.22 & $\mathbf{K} \mathbf{0}$ & 1.485 & $0.016 \pm 0.005$ & CfA \\
\hline J 331 & 11.16 & 1.41 & K9V & 8.495 & 0.0 & GGZG85 \\
\hline$J 316$ & 11.23 & 1.45 & & 367.674 & $0.322 \pm 0.016$ & CfA \\
\hline
\end{tabular}

